feet still showed a large central necrotic zone with healing from the periphery proceeding very slowly (see Fig.).

Presumably, the ulcers on the left acromion and the left outer foot were directly related to the pressure of the full weight of the body. These lesions, however, do not appear to have been nearly as severe

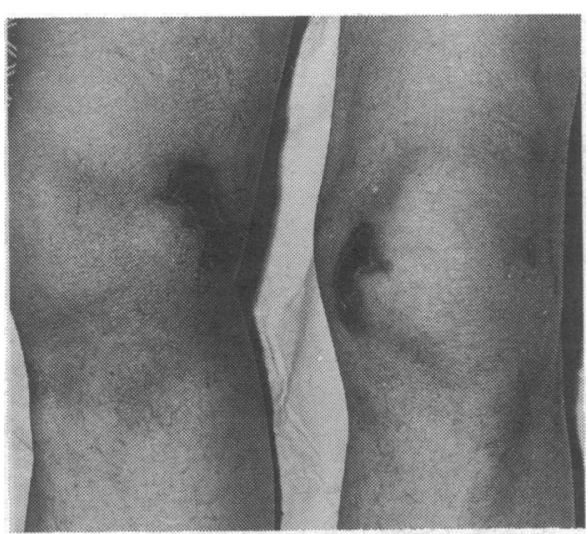

as those on the opposing surfaces of the feet and knees where excessive sweating may have played an important role. It is well known that barbiturates are partly excreted in the sweat and there is a substantial literature quoted by Mandy and Ackerman ${ }^{1}$ which shows that the maximum necrosis in barbiturate blistering is in the eccrine sweat epithelium. Beveridge ${ }^{2}$ has stated that the eccrine sweat glands play a part in the formation of the blisters by excreting barbiturate, the concentration being increased by local blockage such as when two limbs or fingers are in contact. He points out that these are common sites of the bullae rather than the situations of maximal pressure.

The case here reported would tend to confirm that pressure plays some role in barbiturate blistering, but emphasizes that severe epidermal necrosis occurs particularly when pressure and excessive sweat concentration occur in conjunction with each other as in opposing surfaces of limbs which are in close and prolonged contact.

My thanks are due to the department of medical photography of the Oldham Hospitals Group for the photograph.

-I am, etc.,

N. BERLYNE

Oldham and District General Hospital,

Oldham, Lancs

1 Mandy, S., and Ackerman, A. B., Fournal of the American Medical Association, $1970,213,253$.
Beveridge. G. W., Archives of Dermatology,
1970, 101, 369.

STR,-You recently published my paper on Acute Poisoning: Some Myths and Misconceptions (6 March, p. 519), in which I drew attention to the ease with which the contents of articles inadequately supported by laboratory evidence might enter the mythology of acute poisoning. A plea was made to editors and their expert advisers not to publish articles where the sole evidence for the drug ingested was unconfirmed statements by patients or relatives. There could hardly be a better example of this uncritical approach than the paper "Bullous Lesions in Nitrazepam Overdosage" (3 July, p. 28). The title firmly states that bullae occur in nitrazepam overdosage, yet the evidence for in- criminating nitrazepam and excluding barbiturates is no more than vague supposition and conjecture. No mention is made of any laboratory testing for nitrazepam or other drugs.

The only supporting evidence to the occurrence of bullae in nitrazepam overdose is given as " $R$. Goulding (personal communication 1970) at the Poisons Reference Service of Guy's Hospital." In December 1969 I had a communication from Dr. R. Goulding stating that bullae could occur in nitrazepam overdosage. Inquiry as to the validity of this information revealed that other substances might have contributed to the lesions attributed to nitrazepam.

It is not as if nitrazepam cannot be identified in the laboratory. Surely positive laboratory identification of nitrazepam, and negative evidence in the blood or urine regarding barbiturates, methaqualone, meprobamate, glutethimide, opiates, tricyclic antidepressants, and carbon monoxide should have been adduced to substantiate what can now remain but a myth. - I am, etc.,

\section{HenRy Matthew}

Regional Poisoning Treatment Centre, Royal Infirmary, \section{Edinburgh}

\section{Smoking, Lung Cancer, and Asthma}

SIR,-I would like to draw the attention of readers to the possible relationship between smoking, bronchogenic carcinoma, and asthma, because a preliminary survey of a large number of asthma sufferers has suggested some quite fascinating findings on this subject.

After suggestions that certain types of new growth seem to be less prevalent in patients suffering from allergic diseases, an analysis of over 25.000 allergic patients in Australia was started to try to confirm or otherwise - this postulate. This group included some 13,000 asthma sufferers of all types seen over the past 12 years.

Every asthmatic at the first consultation is informed in no uncertain terms that he must give up smoking because of the sensitivity of his respiratory tract. Practically all follow this advice if it is put to them firmly enough; in any event most know from experience that they just cannot smoke without bringing on an attack of wheezing. The number of patients with this disease who are smokers therefore, even to moderate degree, is quite small. Perhaps this is one reason why so few asthma sufferers, even those with severe recurren disease over many years, develop destructive lung lesions such as emphysema as opposed to the chronic bronchitic type of patient, who often uses the excuse that he must continue smoking in order to help him cough up sputum.

The main point of this letter, however, is to record the extraordinary fact that the results of observations so far on these 13,000 true asthma sufferers have shown that only one developed primary bronchogenic carcinoma over this 12-year period, and that was a man who had been informed some five years previously that he just had to give up smoking his 30-odd cigarettes a day, but he did not do so.

These findings suggest two possibilities. Firstly, that patients with asthma may have some resistance to the development of new growth, perhaps as a result of their hyper- sensitive state, or secondly, that patients who do not smoke or give up smoking, even if they have chronic chest diseases, will not develop primary bronchial cancer.

It is hoped to publish the results of this survey in greater detail in the near future, relating them to the number of years each patient has been followed up, etc. It must be stressed at this stage that these findings are preliminary. They would appear to be so significant, however, that they should really be brought to the attention of the medical profession at this early stage because they suggest that further studies of this nature could well be carried out in different areas on an even wider basis.

This letter is largely written because I would be most interested to hear of the opinions of experiences on this subject of any physicians who have had wide experience in the care of bronchial asthma and lung cancer.-I am, etc.,

R. MUNRO FORD

Adelaide,

South Australia

\section{Glutaraldehyde as a Bactericidal Agent}

SIR,-Glutaraldehyde, a dialdehyde, is of particular importance in the field of hospital disinfection because, unlike most other commonly used disinfectants, it possesees sporicidal activity. It is used extensively as a chemosterilizer for endoscopes, anaesthesic equipment, and urological and other surgical instruments. ${ }^{1}$ A stock $2 \%$ solution, when activated with $0.3 \%$ sodium bicarbonate, has a limited shelf-life of two weeks. This deterioration in bactericidal activity is thought to be the result of aldehyde polymerization at alkaline $\mathrm{pH}$, the monomer being considered as being the active form. ${ }^{2}$

Recently, in the course of our studies ${ }^{3}$ into the nature of the antibacterial action of this dialdehyde apainst washed suspensions of Escherichia coli N.C.T.C. 9001 , it was necessary to use different grades of glutaraldehyde obtained from different sources. These grades were: Kotak (25\% solution), KochLight (25\%), Cidex (2\%). Polaron ( $8 \%)$ and Sigma $(50 \%)$. Kodak and Koch-Light are commercial solutions of general industrial application, Cidex is the chemosterilizer used in hospitals, Polaron is a vacuum-distilled preparation containing a high amount of the monomer, and the Sigma preparation is stored over barium carbonate to preven polymerization. Each was diluted to a concentration of $0.01 \%$, sodium bicarbonate added to give a concentration of $0.3 \%$, and $E$. coli incorporated to give an initial count of $3 \times 10^{6}$ viable cells $/ \mathrm{ml}$. Samples were removed at intervals during subsequent incubation for 1 hour at room temperature and viable counts made by the pour-plate method; colonies were counted after incubation of the plates for 48 hours at $37^{\circ} \mathrm{C}$.

Of the glutaraldehyde samples tested. the Kodak solution produced a complete kill after 10 minutes, Cidex and Koch-Light after 20 minutes, and Sigma after 30 minutes, whereas with the highly purified Polaron sample complete loss of viability was not produced even after a 40-minute contact period. The samples containing the largest amounts of polvmeric glutaraldehyde are thus more bactericidal than the samples containing a higher proportion of the monomer.

Although these results refer to one organism only it is apparent that in order to improve the bactericidal efficiency and shelf 